\title{
COMPLEX EIGENVALUE BOUNDS FOR A SCHRÖDINGER OPERATOR ON THE HALF LINE
}

\author{
FRANCESCO FERRULLI AND ARI LAPTEV
}

\begin{abstract}
We derive some bounds on the location of complex eigenvalues for a family of Schrödinger operators $H_{0, \nu}$ defined on the positive half line and subject to integrable complex potential. We generalise the results obtained in [14] where the operator does not have a Hardy term and also include the analysis for potentials belonging to weighted $L^{p}$ spaces. Some information on the geometry of the complex region which bounds the eigenvalues of the radial Schrödinger multidimensional operator are then recovered.
\end{abstract}

To Vladimir Maz'ya with respect and admiration.

\section{INTRODUCTION}

In this notes we study a problem that was posed by V.Maz'ya regarding the location of complex eigenvalues for a family of Schrödinger operators with a Hardy term defined in $L^{2}(0, \infty)$ and perturbed by a complex potential. The operators in question are defined via the differential expression

$$
H_{0, \nu}: u(x) \rightarrow\left(-\frac{d^{2}}{d x^{2}}+\frac{\left(\nu^{2}-1 / 4\right)}{x^{2}}\right) u(x)
$$

and are subject to Dirichlet boundary condition which are assumed to hold at the origin. The special case $\nu=1 / 2$ has been investigated in [14]. The sharp shape of a possible location of the complex spectrum obtained in [14] gave an example that shows that the variational principle does not work for non-selfadjoint operators.

This paper aims at extending the mentioned above result to a larger class of operators (1.1) with $\nu, \nu \geq 0$. Due to the explicit formula for the Green's function for the operator $H_{0, \frac{1}{2}}$, in [14] the existence of a sharp bound for complex eigenvalues of the perturbed operator was established in terms of elementary functions. In this case any eigenvalue $\lambda=|\lambda| e^{i \theta} \in \mathbb{C} \backslash[0, \infty)$

1991 Mathematics Subject Classification. Primary: 35P15; Secondary: 81Q12, 81Q10. Key words and phrases. Schrödinger operator, complex potential, eigenvalue bounds. 
of the operator $H_{0, \frac{1}{2}}+V(x)$ on the half line subject Dirichlet boundary conditions at origin satisfies

$$
|\lambda|^{1 / 2} \leq \frac{1}{2}\left(\sup _{y \geq 0}\left|e^{i \cot (\theta / 2) y}-e^{-y}\right|\right) \int_{0}^{\infty}|V(x)| d x
$$

for any integrable potential $V(x) \in L^{1}(0, \infty)$.

Comparing the expression (1.2) with the analogous results in [1], [3], [4], valid for the operator $H_{0,1 / 2}$ defined on the whole line, we notice that the additional Dirichlet condition imposed at the origin implies a different shape of the region, where eventually the eigenvalues might arise. Unlikely to what happens in the setting of the whole real line, when the bound is expressed only in terms of the modulus of the complex eigenvalue and where the optimal shape is a disc centred at the origin, in the half line case an implicit dependence of such bound upon the phase of the complex eigenvalue is in place, and it implies a break in the symmetry.

Furthermore, in [14], it is shown that the inequality in (1.2) is realised as equality by a certain particular type of potential, showing the optimality of the result. These potentials are of the form $V(x)=c \delta(x-b)$, where the constants $b \in \mathbb{R}$ and $c \in \mathbb{C}$ can be continuously varied so that the unique eigenvalue which is originated from the perturbed operator describes the continuous line in figure 1 .

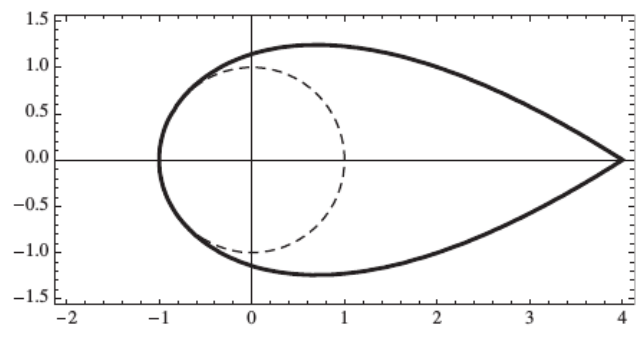

FIGURE 1. Continuous line: the plot of the maximal value of $4|\lambda|$ in the half-line case. Dashed line: the corresponding bound for the Schrödiner operator on the whole line. Ref. [14]

Note that the fact that the region which bounds the complex eigenvalues might depend upon the angle, has been already observed in different context and for the first time in [4]. Other examples are contained in [6], where the operator $H_{0,1 / 2}$ is studied in the Banach space $L^{p}(0, \infty)$ and in [8], where complex perturbations of a second order operator defined on $L^{2}\left(\mathbb{R}^{2}\right)$ are studied, see also [2]. 
One of the corollaries that can be derived from the properties of the complex spectrum of the perturbed operators $H_{0, \nu}, \nu \geq 0$, is about the geometry of the region in the complex plane where eigenvalues of the multidimensional Laplacian can be found when potentials depend only on radius. A first result in this direction, which extends a previous one obtained by Frank for any type of potential [10], was obtained by Frank and Simon in [15]. For some more general cases see [9] and [12]. The authors have proved the validity of the Laptev-Safranov's conjecture [17] for radial potentials and for values of $\gamma \in(0, d / 2)$. In particular, they showed that for dimensions $d \geq 2$ and for any radially symmetric potential $V(|x|)$, any nonpositive eigenvalue of the operator $-\Delta+V(|x|)$ defined on $L^{2}\left(\mathbb{R}^{d}\right)$ satisfies the inequality

$$
|\lambda|^{\gamma} \leq D_{\gamma, d} \int_{\mathbb{R}^{d}}|V(x)|^{\gamma+d / 2} d x=D_{\gamma, d} \int_{\mathbb{R}_{+}}|V(r)|^{\gamma+d / 2} r^{d-1} d r,
$$

where $D_{\gamma, d}$ is a positive constant independent of $V(x)$. Following the ideas adopted in the proof of the formula above we derive, see (3.6), estimates for the eigenvalues of the spectral problem for $H_{0, \nu}$ on the half line of a similar type of (1.3) .

The paper is organised as follows. In Section 2 we extend the results obtained in [14] to arbitrary $\nu \geq 0$. Then assuming $V(x) \in L^{1}(0, \infty)$ we derive uniform bounds for the spectrum and show that also in the general case $\nu \neq 1 / 2$, the region, which bounds the eigenvalues in the complex plane, depends on the phase. In section 3 we deduce similar uniform bounds obtained in Section 2 for potentials belonging to special weighted $L^{p}$ spaces. In particular, using the spherical harmonics reduction of the Laplacian on $\mathbb{R}^{d}$, we observe that the region, where any eigenvalue $\lambda$ generated by a radially symmetric complex potential can be found, depends again on the phase of $\lambda$ and the $d$-dimensional $L^{p}$-norm of the potential.

Note that in [5] the authors considered Lieb-Thirring inequalities for Schrödiner operators with a Hardy term. Some Lieb-Thirring inequalities for Schrödinger operators with complex-valued potentials were studied in [11].

\section{UNIFORM BOUNDS}

Let us define

$$
H:=H_{0, \nu}+V(x), \quad x>0,
$$

where $V(x)$ is a complex-valued potential and let us consider the associated eigenvalue problem

$$
H u=\lambda u \text {. }
$$


In the following we understand $H$ as the operator generated by its respective sesquilinear form. Details on how this construction is realised can be found for example in Section 2 of [17]. We note that the classical Hardy inequality and suitable conditions on $V$, guarantee the boundedness from below of the obtained form. In particular,

$$
\sigma_{\mathrm{ess}}(H)=\sigma_{\mathrm{ess}}\left(H_{0, \nu}\right)=[0, \infty) .
$$

and that the spectrum $\sigma(H)$ of the operator $H$ is discrete in $\mathbb{C} \backslash \sigma\left(H_{0, \nu}\right)$. The Green's function of the operator $H_{0, \nu}$ equals

$$
G_{\nu}(x, y, z)= \begin{cases}\frac{\pi}{2 i} \sqrt{x} H_{\nu}^{(1)}(\sqrt{z} x) \sqrt{y} J_{\nu}(\sqrt{z} y) & \text { if } y \leq x, \\ \frac{\pi}{2 i} \sqrt{x} J_{\nu}(\sqrt{z} x) \sqrt{y} H_{\nu}^{(1)}(\sqrt{z} y) & \text { if } y>x\end{cases}
$$

where $J_{\nu}(z)$ and $H_{\nu}^{(1)}(z)$ are respectively the Bessel and Hankel function of first kind of order $\nu$ (see for example [6] and [22]).

Note that, since $H_{0, \nu}$ is selfadjoint, the Green's function is symmetric in its two variables

$$
G_{\nu}(x, y, z)=G_{\nu}(y, x, z)
$$

Let us introduce the factorization of the potential $V(x)$

$$
V(x)=W_{1}(x) W_{2}(x):=\left(V(x)|V(x)|^{-\frac{1}{2}}\right)|V(x)|^{\frac{1}{2}},
$$

and consider the Birman-Schwinger operator

$$
B(\lambda):=W_{2} R(\lambda) W_{1}
$$

where $R(\lambda)$ stands for the resolvent operator $\left(H_{0}-\lambda\right)^{-1}$. We proceed now customarily by recalling the following correspondence which holds between the eigenvalues of $H$ and those of the Birman-Schwinger operator. It holds that if $\lambda$ is an eigenvalue $H$ then -1 is an eigenvalue of the operator $B(\lambda)$. Therefore a necessary condition for $\lambda$ to be an eigenvalues of the operator (2.1) implies that the inequality

$$
1 \leq\|B(\lambda)\|
$$

Theorem 1. Let $\nu \geq 0$ and let $V(x) \in L^{1}(0, \infty)$ be a complex-valued potential. Assume that $\lambda \in \mathbb{C} \backslash[0, \infty)$ such that $\lambda=|\lambda| e^{i \theta}$ where $\theta \in(0,2 \pi)$, is an eigenvalue of the operator $H_{0, \nu}+V(x)$ with Dirichlet boundary condition at the origin. Then there exists a constant $C(\nu, \theta)$ such that:

$$
|\lambda|^{1 / 2} \leq C(\nu, \theta) \int_{0}^{\infty}|V(x)| d x,
$$

where the constant $C(\nu, \theta)$ depends only on the angle $\theta$ and $\nu$ and equals

$$
C(\nu, \theta)=\frac{\pi}{2} \sup _{0 \leq y \leq x<\infty}\left(\sqrt{x}\left|H_{\nu}^{(1)}\left(x e^{i \theta / 2}\right)\right| \sqrt{y}\left|J_{\nu}\left(y e^{i \theta / 2}\right)\right|\right) .
$$


The key estimates which will be used later in the proof of theorem 1 are the asymptotic behaviour of the Bessel's functions $J_{\nu}(z)$ and $H_{\nu}^{(1)}(z)$ near zero and infinity. We collect them in the following lemma for the reader's convenience and we refer to [19], [7] for the details.

Lemma 1. Let $z \in \mathbb{C}$. Then:

$$
\begin{array}{ll}
J_{\nu}(z) \sim \frac{\left(\frac{1}{2} z\right)^{\nu}}{\Gamma(\nu+1)} & \text { for } z \rightarrow 0 \\
H_{\nu}^{(1)}(z) \sim-\frac{i}{\pi} \Gamma(\nu)\left(\frac{z}{2}\right)^{-\nu} & \text { for } z \rightarrow 0
\end{array}
$$

and

$$
\begin{aligned}
& J_{\nu}(z) \sim \sqrt{\frac{2}{\pi}} \sqrt{\frac{1}{z}}\left(\cos \left(z-\frac{\nu \pi}{2}-\frac{\pi}{4}\right)+e^{|\Im z|} O(1)\right) \quad \text { for } z \rightarrow \infty, \\
& H_{\nu}^{(1)}(z) \sim \sqrt{\frac{2}{\pi}} \sqrt{\frac{1}{z}} e^{i\left(z-\frac{\nu \pi}{2}-\frac{\pi}{4}\right)} \quad \text { for } z \rightarrow \infty,
\end{aligned}
$$

Proof of the Theorem 1. The proof is standard and uses the idea from [1] and [14]. In the first instance we will consider the spectral parameter $\lambda$ lying on the unitary complex circle. Let $s=\lambda=e^{i \theta}$ where $\theta \in(0,2 \pi)$. By mean of the Schur's inequality, we estimate the operator norm of $B(\lambda)$ as

$$
\begin{aligned}
\mid \iint B(\lambda) & \bar{f}(x) g(y) d y d x \mid \\
& \leq\left(\iint\left|\bar{W}_{2}(x)\right|^{2}\left|G_{\nu}(x, y, s)\right||g(y)|^{2} d y d x\right)^{1 / 2} \\
& \left(\iint\left|\bar{W}_{1}(y)\right|^{2}\left|G_{\nu}(x, y, s)\right||f(x)|^{2} d y d x\right)^{1 / 2} \\
& \leq \sup _{x \in(0, \infty)} \int_{0}^{\infty}|V(y)|\left|G_{\nu}(x, y, s)\right| d y\|f\|_{L^{2}}\|g\|_{L^{2}} \\
& \leq \sup _{x, y \in(0, \infty)}\left|G_{\nu}(x, y, s)\right| \int_{0}^{\infty}|V(y)| d y\|f\|_{L^{2}}\|g\|_{L^{2}} \quad \text { (2.8) }
\end{aligned}
$$

As previously observed, the Green's function $G_{\nu}(x, y, s)$ is symmetric in its variable $x$ and $y$. Therefore it will be sufficient to consider the above 
supremum in the sector $0 \leq y \leq x<\infty$. Thus, we define the constant

$$
\begin{aligned}
C(\nu, \theta)=\sup _{0 \leq y \leq x<\infty} & \left|G_{\nu}(x, y, s)\right| \\
& =\frac{\pi}{2} \sup _{0 \leq y \leq x<\infty}\left(\sqrt{x}\left|H_{\nu}^{(1)}\left(x e^{i \theta / 2}\right)\right| \sqrt{y}\left|J_{\nu}\left(y e^{i \theta / 2}\right)\right|\right) .
\end{aligned}
$$

The finiteness of the constant $C(\nu, \theta)$ comes immediately from the regularity of the Bessel's function and from the asymptotics stated in (2.6) and (2.7). Let us consider now the general case, when the spectral parameter $\lambda \in \mathbb{C} \backslash[0, \infty]$ is given by $\lambda=|\lambda| e^{i \theta}$. From rescaling we immediately deduce that

$$
\sup _{0 \leq y \leq x<\infty} \frac{\pi}{2} \sqrt{x}\left|H_{\nu}^{(1)}(x \sqrt{\lambda})\right| \sup _{y \in(0, x)} \sqrt{y}\left|J_{\nu}(y \sqrt{\lambda})\right|=\frac{C(\nu, \theta)}{|\lambda|^{1 / 2}}
$$

and this complete the proof of (2.4). We remark here that, on the opposite of what happen in the case $\nu=1 / 2$, the angle dependence is also important in the definition of the best constant. We note that for small values of the angle $\theta$ and for $\nu>1 / 2$, the supremuum of the function $\sqrt{y}\left|J_{\nu}(y s)\right|$ for $y \in(0, x)$ is in general not attained at the point $y=x$ as it happens in the case $\nu=1 / 2$ for all the angle values. This phenomenon can be easily understood by the fact that the Bessel function $J_{\nu}(x)$ with real argument is a bounded oscillating function and such oscillatory behaviour is still present for small values of $\theta$ when $J_{\nu}\left(|\lambda| e^{i \theta} x\right)$.

For what concern the sharpness in the case $V(x)=c \delta(x-b)$, we proceed by standard arguments. Let us consider $u(x)$ solution of

$$
H_{0, \nu} u(x)+c \delta(b-x) u(x)=\lambda u(x) .
$$

Let $c_{1}$ and $c_{2}$ be two complex constants and write

$$
\begin{array}{ll}
u(x)=\sqrt{x} H_{\nu}^{(1)}(\sqrt{\lambda} x) & \text { for } x>b, \\
u(x)=c_{1} \sqrt{x} J_{\nu}(\sqrt{\lambda} x)+c_{2} \sqrt{x} H_{\nu}^{(1)}(\sqrt{\lambda} x) & \text { for } x<b .
\end{array}
$$

We first impose the Dirichlet boundary condition at zero and the continuity at point $b$ for $u(x)$, which immediately yields

$$
c_{1}=\frac{H_{\nu}^{(1)}(\sqrt{\lambda} b)}{J_{\nu}(\sqrt{\lambda} b)}, \quad c_{2}=0
$$

The following condition that has to be imposed is the discontinuity jump at point $b$ for the derivatives, namely

$$
\left[c_{1} \frac{d}{d x}\left(\sqrt{x} J_{\nu}(\sqrt{\lambda} x)\right)-\frac{d}{d x}\left(\sqrt{x} H_{\nu}^{(1)}(\sqrt{\lambda} x)\right)\right]_{(x=b)}=c H_{\nu}^{(1)}(\sqrt{\lambda} b)
$$


which by means of the formulae for the Wronskian of Bessel's functions, reduces to

$$
\frac{2 i \sqrt{\lambda}}{\pi}=c H_{\nu}^{(1)}(\sqrt{\lambda} b) J_{\nu}(\sqrt{\lambda} b)
$$

Setting $c=|c| e^{i \phi}$, one can first determine $b$ such that

$$
\frac{\pi}{2}\left|H_{\nu}^{(1)}(\sqrt{\lambda} b) J_{\nu}(\sqrt{\lambda} b)\right|=C(\nu, \theta)
$$

and then derive the phase of $c$ from equation (2.10).

Numerical computations of $C(\nu, s)$ show a qualitative accordance for different values of the order $\nu$ and the case $\nu=1 / 2$.

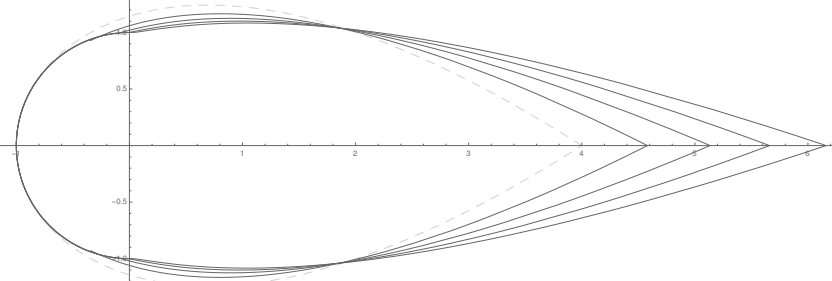

FIGURE 2. Continuous lines: plot of the numerical approximation value of the constant $C(\nu, \theta)$ in (2.5) as function of the angle, for different values of $\nu=1,3 / 2,2,5 / 2$. The dashed line is the case $\nu=1 / 2$ and plotted as reference.

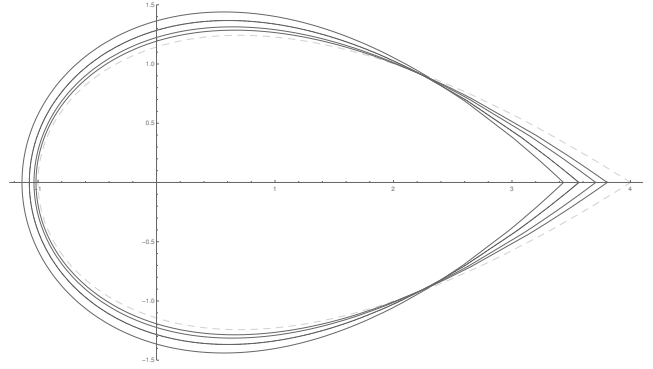

FIGURE 3. Continuous lines: plot of the numerical approximation value of the constant $C(\nu, \theta)$ in (2.5) as function of the angle, for different values of $\nu=1 / 3,1 / 4,1 / 8,1 / 250$. The dashed line is the case $\nu=1 / 2$ and plotted as reference.

Remark 1. We note that in the special case $\nu=1 / 2$, the constant $C(\nu, s)$ has an explicit formulation which is given in (1.2). 


\section{THE CASE $V(x) \in L^{p}$}

In this section we suggest a different estimate type from the one used in the previous section for the quantity at the beginning of the inequality chain (2.8). This in turn allows us to derive estimates valid for potentials in weighted $L^{p}(0, \infty)$ spaces; the weights, which might at first look artificial, are in fact recovered from the analysis done in [15].

We proceed denoting by $\tilde{G}_{\nu}(x, s)$ the quantity $\tilde{G}_{\nu}(x, s):=$ $\sup _{y \in(0, \infty)}\left|G_{\nu}(x, y, s)\right|$

$$
\begin{array}{r}
\tilde{G}_{\nu}(x, \theta)=\frac{\pi}{2} \max \left(\sup _{y \in(0, x)} \sqrt{x}\left|H_{\nu}^{(1)}\left(x e^{\theta / 2}\right)\right| \sqrt{y}\left|J_{\nu}\left(y e^{\theta / 2}\right)\right|,\right. \\
\left.\sup _{y \in(x, \infty)} \sqrt{y}\left|H_{\nu}^{(1)}\left(y e^{\theta / 2}\right)\right| \sqrt{x}\left|J_{\nu}\left(x e^{\theta / 2}\right)\right|\right),
\end{array}
$$

where $\theta \in(0,2 \pi)$. Let us now introduce a weight function $w(x)$ such that

$$
\begin{aligned}
\int_{0}^{\infty}|V(x)| \tilde{G}_{\nu}(x, s) d x \leq & \left(\int_{0}^{\infty}(w(x)|V(x)|)^{\frac{d}{2}+\gamma} d x\right)^{\frac{2}{d+2 \gamma}} \\
& \left(\int_{0}^{\infty} \tilde{G}_{\nu}(x, s)^{\beta} w(x)^{-\beta} d x\right)^{\frac{1}{\beta}}
\end{aligned}
$$

where $\beta$ satisfies $\left(\gamma+\frac{d}{2}\right)^{-1}+\beta^{-1}=1$. We furthermore assume the weight function to be a homogeneous function of degree $\alpha$. For this reason let us introduce $w(x)=x^{\alpha}$ for any positive $\alpha$ such that $\alpha\left(\frac{d}{2}+\gamma\right)=d-1$. This, together with the previous assumption made on $\beta$ gives the following values

$$
\alpha=2(d-1) /(d+2 \gamma)
$$

and

$$
\beta=\frac{d+2 \gamma}{d+2 \gamma-2}
$$

Let us finally introduce the constant $\tilde{C}(\nu, \theta)$ as

$$
\tilde{C}(\nu, \theta):=\left(\int_{0}^{\infty} \tilde{G}_{\nu}(x, \theta)^{\beta} w(x)^{-\beta} d x\right)^{\frac{1}{\beta}} .
$$

We can now formulate the main results of this section in the following theorem.

Theorem 2. Let $\nu>0$ and $\lambda \in \mathbb{C} \backslash[0, \infty)$ such that $\lambda=|\lambda| e^{i \theta}$ where $\theta \in(0,2 \pi)$. Let $\lambda$ be a complex eigenvalue of the operator $H_{0, \nu}+V(x)$ with Dirichlet boundary condition at the origin and where $V(x) \in L^{d / 2+\gamma}(0, \infty)$ 
for any real value of $d \geq 2(1-\gamma)$ such that $0<\gamma<d / 2$. Then there exists a constant $\tilde{C}(\nu, \theta)$ such that

$$
|\lambda|^{\gamma} \leq \tilde{C}(\nu, \theta)^{\frac{d}{2}+\gamma} \int_{0}^{\infty} x^{d-1}|V(x)|^{\frac{d}{2}+\gamma} d x,
$$

where the constant $\tilde{C}(\nu, \theta)$, which depends upon the angle $\theta$ and the order $\nu$, is defined in (3.5).

Proof. From the properties of the Bessel functions (2.6) and (2.7), we deduce that the function $\tilde{G}_{\nu}(x, s)$ defined in (3.1) is a bounded function on $x \in(0, \infty)$ which converges to zero at the origin with order one

$$
\begin{aligned}
& \tilde{G}_{\nu}(x, s) \sim O(x) \quad \text { for } x \rightarrow 0, \\
& \tilde{G}_{\nu}(x, s) \sim O(1) \quad \text { for } x \rightarrow \infty \text {. }
\end{aligned}
$$

Therefore, the condition $0<\gamma<d / 2$ provides the convergence of the integral in the second factor of right hand side of (3.2). From a homogeneity argument, it follows that for general $\lambda \in \mathbb{C}$ we have

$$
\left(\int_{0}^{\infty} \tilde{G}_{\nu}(x, \lambda)^{\beta} w(x)^{-\beta} d x\right)^{\frac{1}{\beta}}=\frac{\tilde{C}(\nu, s)}{|\lambda|^{\frac{1-\alpha}{2}+\frac{1}{2 \beta}}},
$$

and so the proof is complete.

Remark 2. Note that the condition $d \geq 2(1-\gamma)$ is essential in our type of analysis in order to use the Holder inequality. In the special case of $d=1$, then $\gamma$ is allowed to take also the value $d / 2$. With the notation of the theorem we have $\alpha=0$ and $\beta=\infty$, which is the result in theorem 1

For fixed $d$, numerical evidences suggest not only that the contour of the region $\mathcal{S}_{d, \nu}$, where the complex eigenvalues might lie, changes varying the angle in the complex plane, but according to what would happen for a real perturbation, for greater values of $\nu$ the region $\mathcal{S}_{d, \nu}$ obtained shrinks, concentrating around the origin. It seems, in fact, the case that the regions appear to be ordered in a monotonic inclusion order $\mathcal{S}_{d, \nu^{\prime}} \subseteq \mathcal{S}_{d, \nu}$ for $\nu<$ $\nu^{\prime}$, as shown in Figure 4 and Figure 5 below. In particular the estimates obtained above for the half line problems provide an important insight on the shape of the region $\mathcal{S}_{d}^{r}$ where the complex eigenvalues of the Laplacian with complex radial perturbation and restricted to radial functions might lie.

Let us consider the $d$-dimensional Laplacian in polar coordinates

$$
\Delta_{d} f=\frac{1}{r^{(d-1)}} \frac{\partial}{\partial r}\left(r^{(d-1)} \frac{\partial f}{\partial r}\right)+\frac{1}{r^{2}} \Delta_{S^{(d-1)}} f
$$




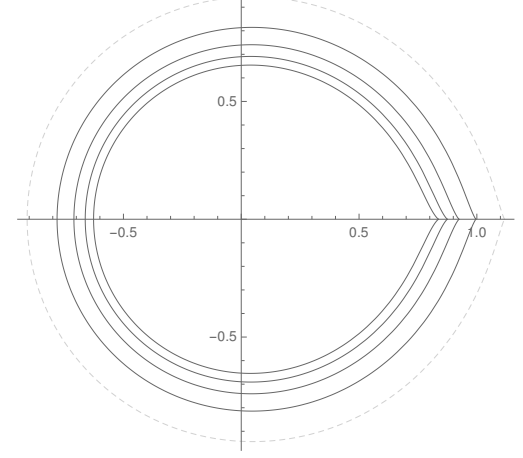

FIGURE 4. Continuous lines: from the outer most, plots of the numerical approximation value of the constant $\tilde{C}(\nu, \theta)$ as function of the angle, for different values of $\nu=$ $1,3 / 2,2,5 / 2$ in (3.6), with $\gamma=1 / 2$ and $d=3$. The dashed line corresponds to the case $\nu=1 / 2$.

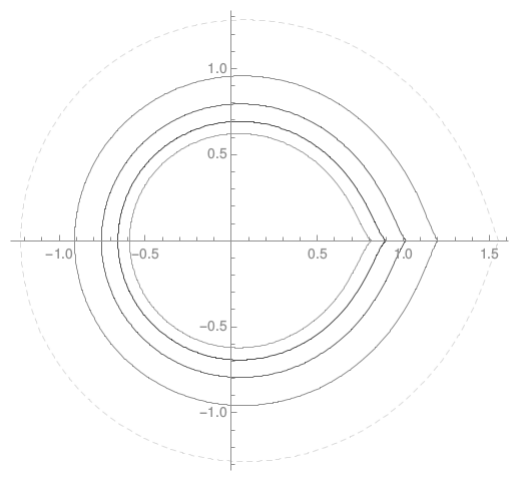

FIGURE 5. Continuous lines: from the outer most, plots of the numerical approximation value of the constant $\tilde{C}(\nu, \theta)$ as function of the angle, for different values of $\nu=$ $1,3 / 2,2,5 / 2$ in (3.6), with $\gamma=1 / 2$ and $d=5$. The dashed line corresponds to the case $\nu=1 / 2$.

where $\Delta_{S^{(d-1)}}$ is the Laplace-Beltrami operator on the unitary $(d-1)$-sphere $S^{(d-1)}$. It is a well known fact that the eigenspaces $E_{l}$ of the LaplaceBeltrami operator on the $d$-sphere, with $l=0,1,2, \ldots$ corresponding to the eigenvalue $c_{l, d}:=l(l+d-2)$ yield the following direct sum decomposition

$$
L^{2}\left(S^{(d-1)}\right)=\bigoplus_{l=0}^{\infty} E_{l}^{d}
$$

where $E_{l}$ are close, pairwise orthogonal and such that for each of them there exists a basis of orthonormal spherical harmonics 
$Y_{l, m}$ where the index $m$ runs on an appropriate set of finite cardinality which depends on $l$. We observe that the subspaces $\tilde{E}_{l}=\left\{f(x)=R(r) Y_{l, m}(\omega) \mid r \in(0, \infty), \omega \in S^{(d-1)}\right\}$ reduces the operator $-\Delta+V(|x|)$. It follows that

$$
-\Delta_{d}+V(|x|)=\bigoplus_{l, m} \tilde{h}_{l}^{d}
$$

where

$$
\tilde{h}_{l}^{d}:=-\frac{\partial^{2}}{\partial r^{2}}-\frac{(d-1)}{r} \frac{\partial}{\partial r}+\frac{l(l+d-2)}{r^{2}}+V(|x|),
$$

with $\mathcal{D}\left(\tilde{h}_{l}^{d}\right)=L^{2}\left((0, \infty), r^{\xi} d r\right)$ and $\xi=\left(\frac{d-1}{2}\right)$. By mean of the unitary transformation $R(r) \rightarrow u(r)=r^{\xi} R(r)$ (see [21] for the case $d=3$ ) and from the definition of $H_{0, \nu}$ given in (1.1), the above operators simplifies to

$$
h_{l}^{d}:=H_{0, N(d, l)}+V(|x|), \quad \mathcal{D}\left(\tilde{h}_{l}\right) \subseteq L^{2}(0, \infty),
$$

where

$$
N(d, l)=\left|\sqrt{c_{l, d}+\xi(\xi-1)+1 / 4}\right| .
$$

It finally follows the decomposition of the spectrum of the operator $-\Delta_{d}+$ $V(|x|)$ in terms of

$$
\sigma\left(-\Delta_{d}+V(|x|)\right)=\overline{\bigcup_{l} \sigma\left(h_{l}^{d}\right)}
$$

From the property $\mathcal{S}_{d, \nu^{\prime}} \subseteq \mathcal{S}_{d, \nu}$ for $\nu<\nu^{\prime}$, we in fact conclude that

$$
\sigma\left(-\Delta_{d}+V(|x|)\right) \subseteq \mathcal{S}_{d, N(d, 0)}
$$

which in turn implies that the shape of the region $\mathcal{S}_{d}^{r}$ where the complex eigenvalues of the Laplacian with complex radial perturbation and restricted to radial functions lie, depend in fact on the phase of the eigenvalue itself. In particular, for $d=3$ the shape of $\mathcal{S}_{3}^{r}$ is determined by the contour corresponding the value $\nu=1 / 2$ in Figure 4 , and for $d=5$ by the one corresponding to $\nu=3 / 2$ in Figure 5. In general, we observe, it holds $N(d, 0)=\frac{d-2}{2}$. 


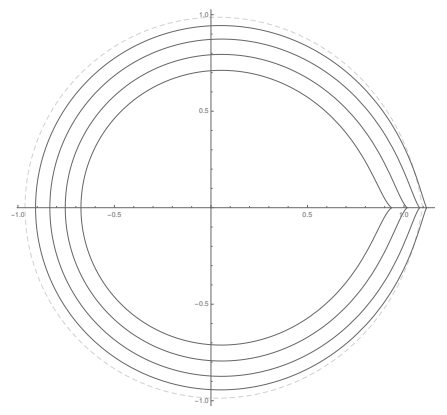

FIGURE 6. Continuous lines: contour of the regions $\mathcal{S}_{d}^{r}$ for $d=3,4,5,6$ obtained respectively for the values $\nu=$ $1 / 2,1,3 / 2,2$. The dashed line is an approximation to the limit case $d=2$ and $\nu=0$.

\section{REFERENCES}

[1] A. A. Abramov, A. Aslanyan, and E. B. Davies, Bounds on complex eigenvalues and resonances. J. Phys. A, (1999), no. 2, pp. 57-72.

[2] J-C. Cuenin, A. Laptev and C. Tretter, Eigenvalue Estimates for NonSelfadjoint Dirac Operators on the Real Line. AHP, 15 (2014), 707-736.

[3] E.B. Davies. Non-self-adjoint differential operators. Bulletin of the London Mathematical Society, 34 (2002), no. 5, 513-532.

[4] E. B. Davies and Jiban Nath, Schrödinger operators with slowly decaying potentials Journal of computational and applied mathematics. 148 (2002), no. 1, $1-28$.

[5] T. Ekholm and R.L. Frank, Lieb-thirring inequalities on the half-line with critical exponent. J. Eur. Math. Soc., 10 (2008), no. 3, 739-755.

[6] A. Enblom, Estimates for eigenvalues of schrdinger operators with complexvalued potentials. Letters in Mathematical Physics, 106 (2016), no. 2, 197-220.

[7] W.N. Everitt and H. Kalf, The Bessel differential equation and the Hankel transform. Journal of Computational and Applied Mathematics, 208 (2007), no.1, 3-19.

[8] F. Ferrulli, A. Laptev and O. Safronov, Eigenvalues of the bilayer graphene operator with a complex valued potential. Analysis and Mathematical Physics, (2018), DOI 10.1007/s13324-018-0262-4.

[9] R.L. Frank, Eigenvalue bounds for Schrödinger operators with complex potentials. III.. Transactions of the American Mathematical Society, 307 (2018), no. 1, 219-240.

[10] R.L. Frank, Eigenvalue bounds for Schrödinger operators with complex potentials. Bulletin of the London Mathematical Society, 43 (2011), no. 4, 745-750.

[11] R.L. Frank, A. Laptev, E.H. Lieb and R. Seiringer, Lieb-Thirring inequalities for Schrödinger operators with complex-valued potentials. Letters in Mathematical Physics, 77 (2006), no. 3, 309-316.

[12] R.L. Frank and J. Sabin, Restriction theorems for orthonormal functions, Strichartz inequalities and uniform Sobolev estimates. Amer. J. of Math. 139 (2017), no. 6, 1649-1691. 
[13] ] R.L. Frank, A. Laptev and O. Safronov, On the number of eigenvalues of Schrödinger operators with complex potentials. JLMS, 94 (2016), no. 2, 377390.

[14] R.L. Frank, A. Laptev and R. Seiringer, A sharp bound on eigenvalues of Schrödinger operators on the half-line with complex-valued potentials. In: Spectral Theory and Analysis, J. Janas et al. (eds.), 39 - 44, Oper. Theory Adv. Appl. 214, BirkhŁuser, Basel, 2011.

[15] R. Frank and B. Simon, Eigenvalue bounds for Schrödinger operators with complex potentials, II., J. Spectr. Theory, 7 (2017), no. 3, 633-658.

[16] J.B. Keller, Lower bounds and isoperimetric inequalities for eigenvalues of the Schrödinger equation. Journal of Mathematical Physics, 2 (1961), no. 2, 262-266.

[17] A. Laptev and O. Safronov, Eigenvalue estimates for Schrödinger operators with complex potentials. Communications in Mathematical Physics, 292 (2009), no. 1, 29-54.

[18] H. Mizutani, Eigenvalue bounds for non-self-adjoint Schrödinger operators with the inversesquare potential. arXiv preprint arXiv:1607.01727, 2016.

[19] F.W.J. Olver, NIST handbook of mathematical functions hardback and CDROM. Cambridge university press, 2010.

[20] O. Safronov, Estimates for eigenvalues of the Schrödinger operator with a complex potential. Bulletin of the London Mathematical Society, 42 (2010), no. 3, 452-456.

[21] G. Teschl. Mathematical methods in quantum mechanics. Graduate Studies in Mathematics, 99, 2009.

[22] E.C. Titchmarsh, Eigenfunction expansions associated with second-order differential equations, Part 1. Oxford University Press, 1962.

Francesco Ferrulli, Department of Mathematics, Imperial College LONDON, SW7 2AZ, LONDON, UK

E-mail address: f.ferrullilu@imperial.ac.uk

Ari laptev, Department of Mathematics, imperial College london, SW7 2AZ, London, UK, Institut Mittag-LefFler and SAint Petersburg UNIVERSITY

E-mail address: a. laptev@imperial.ac.uk 